\title{
Metallized hyperbranched polydiyne: a photonic material with a large refractive index tunability and a spin-coatable catalyst for facile fabrication of carbon nanotubes $\dagger$
}

\author{
Matthias Häußler, ${ }^{a}$ Jacky Wing Yip Lam, ${ }^{a}$ Anjun Qin, ${ }^{a b}$ Kalvin Ka Chun Tse, ${ }^{a}$ Martin Ka Shing Li, ${ }^{c}$ \\ Jianzhao Liu, ${ }^{a}$ Cathy Ka Wai Jim, ${ }^{a}$ Ping $\mathrm{Gao}^{c}$ and Ben Zhong Tang*ab
}

Received (in Cambridge, UK) 26th February 2007, Accepted 21st March 2007

First published as an Advance Article on the web 2nd April 2007

DOI: $10.1039 / b 702915 b$

A cobalt-containing hyperbranched polydiyne shows refractive indexes ( $n$ ) as high as 1.713-1.813 in the long wavelength region, which can be tuned to a large extent ( $\Delta n$ up to $\sim 0.048$ ) by UV irradiation; the polymer can also function as a spin-coatable catalyst for the growth of carbon nanotubes.

Organometallic polymers are molecular hybrids of organic and metallic species that exhibit useful catalytic, photonic, electrical, optical and magnetic properties as well as excellent macroscopic processability, which are often inaccessible by their pure organic and inorganic counterparts. ${ }^{1-3}$ Polymers containing lead and iron atoms in the polymer backbones, for example, exhibit refractive index (RI) values higher than those of their organic cousins. ${ }^{4,5}$ Development of new, processable materials with high RI values is fascinating yet rather challenging. To find practical applications in optoelectronic devices such as optical waveguides, memories, and photochromatic and holographic image recording systems, the highly refractive materials should ideally undergo large changes in their refractivities when stimulated by an external perturbation. ${ }^{6}$

Our group has been interested in the design and synthesis of hyperbranched conjugated polymers. ${ }^{7}$ We have succeeded in the preparation of completely soluble hyperbranched polydiynes ( $h b$ PDYs) with high molecular weights. The internally triple-bonded scaffolds of the $h b$-PDYs function as excellent macroligands for complexation with metallic species such as cobalt carbonyls. The cobalt-containing $h b$-PDYs have been transformed into advanced ceramics, which exhibit outstanding soft ferromagnetism with very high magnetizability $\left(M_{\mathrm{s}}\right.$ up to $\sim 120 \mathrm{emu} \mathrm{g}^{-1}$ ) and extremely low hysteresis losses $\left(H_{\mathrm{c}}\right.$ and $M_{\mathrm{r}}$ approaching zero). ${ }^{8,9}$ In this short communication, using a cobalt-metallized, triphenylamine-cored $h b$-PDY as an example, we show that the organometallic $h b$-PDYs possess high RIs in the long wavelength region, which can be modulated to a large extent by photolysis. We also demonstrate that spin-coated thin films of the metal-polymer hybrids can serve as catalyst seeds for the fabrication of carbon nanotubes (CNTs)

${ }^{a}$ Department of Chemistry, The Hong Kong University of Science \& Technology (HKUST), Clear Water Bay, Kowloon, Hong Kong, China. E-mail:tangbenz@ust.hk; Tel: +852-2358-7375

${ }^{b}$ Department of Polymer Science and Engineering, Zhejiang University, Hangzhou 310027. China

${ }^{c}$ Department of Chemical Engineering, HKUST, Hong Kong, China

$\uparrow$ Electronic supplementary information (ESI) available: Detailed experimental procedures and FT-IR spectra of the thin solid films of $h b$ PTEPA(Co) before and after UV irradiation. See DOI: 10.1039/b702915b
The $h b$-PDY used in this study is poly[tris(4-ethynylphenyl)amine] ( $h b$-PTEPA), which is readily metallized, through its facile complexation with octacarbonyldicobalt, to give $h b$-PTEPA(Co) (Scheme 1). ${ }^{8,9}$ Spin-coating toluene solutions of $h b$-PTEPA(Co) onto silicon wafers or glass substrates gives thin films of good quality with thickness of $\sim 1 \mu \mathrm{m}$, as determined by ellipsometry measurement. The films of $h b$-PTEPA(Co) are stable and can be stored without any special protection under ambient conditions for more than six months. UV irradiation $(\lambda=360-400 \mathrm{~nm})$, however, can induce a visible photochromic response, readily bleaching the brownish films.

The color change may stem from photoinduced decomplexation of the organocobalt polymer. Fig. S1 shows the IR spectra of the films of $h b$-PTEPA(Co) before and after UV irradiation. Whereas $h b$-PTEPA(Co) shows strong, typical cobalt-carbonyl absorptions at 2090, 2055 and $2025 \mathrm{~cm}^{-1},{ }^{10}$ these bands are greatly weakened by UV irradiation. The bands at 569,515 and $494 \mathrm{~cm}^{-1}$, associated with cobalt-carbon vibrations and cobalt-carbonyl deformations, ${ }^{11}$ almost completely disappear after the photolysis. On the other hand, a broad shoulder emerges in the $\mathrm{C}=\mathrm{C}$ stretching region at $1700-1600 \mathrm{~cm}^{-1}$, suggesting that diene, triene and/or higher conjugated double-bond structures have resulted from the photoinduced polymerization of the diyne moieties after metal decomplexation of $h b$-PTEPA $(\mathrm{Co})$. Other absorption bands remain more or less the same, further confirming that the photobleaching originates from the decomplexation of the organometallic moieties.

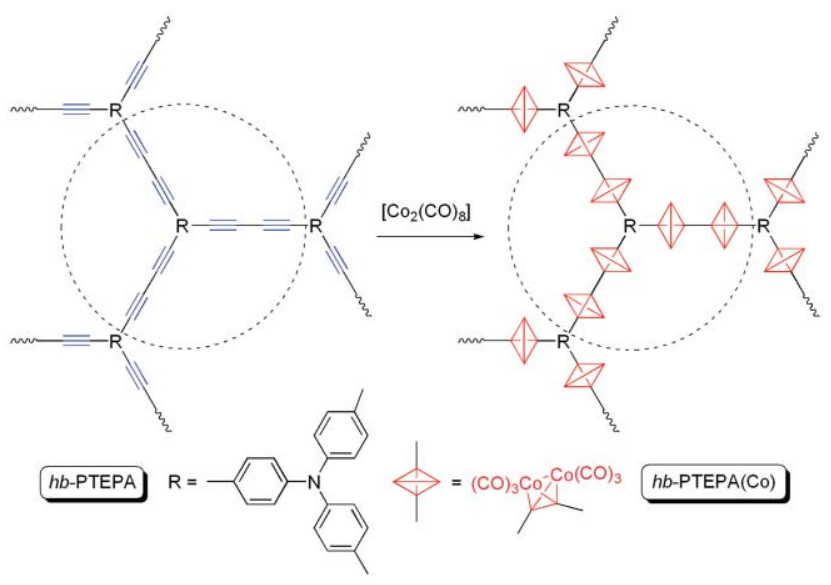

Scheme 1 Preparation of cobalt-containing polymer $h b$-PTEPA(Co) via metallization of hyperbranched polydiyne $h b$-PTEPA. 
We utilized the visible photochromic change to fabricate microstructured films. Photopatterns are readily generated by simply irradiating a thin film of $h b$-PTEPA(Co) with UV light for $30 \mathrm{~min}$ through a Cu-negative mask, without going through a wet or dry development step. Fig. 1A shows an optical micrograph of the photopatterned film, with the bleached areas being those exposed to the UV light. Fig. 1B shows a magnified image where sharp edges between the exposed and unexposed regions are clearly seen, while Fig. 1C illustrates the excellent patternability of the polymer film. The feature size of the photonic pattern is basically determined by the photomask used. We believe that smaller feature sizes should be achievable by employing alternative patterning techniques such as electron-beam lithography and multi-beam interference holography. ${ }^{12}$

Wavelength-dependent RI measurements of the unexposed and exposed films of $h b$-PTEPA $(\mathrm{Co})$ reveal interesting results. Like its nonmetallated parent $(h b$-PTEPA $),{ }^{8}$ the metallated polymer exhibits very high RIs $(n=1.813-1.713)$ in the spectral region of 600-1700 nm (Fig. 2). Its RIs drop dramatically after the UV irradiation ( $n=1.777-1.665$ ), possibly due to the large structural changes induced by the photolysis of the organometallic complex. The difference in the RI $(\Delta n)$ at the telecommunication-important wavelength $(\lambda=1550 \mathrm{~nm})$ is as large as 0.047 (Table 1).

For a material to be useful for technological applications, its optical dispersion, which is a measure of the change in its RI with wavelength, should be small. For a conventional optical material working in the visible spectral region, its optical dispersion is expressed by the Abbé number $\left(v_{\mathrm{D}}\right)$, which is calculated from equation 1:

$$
v_{\mathrm{D}}=\frac{n_{\mathrm{D}}-1}{n_{\mathrm{F}}-n_{\mathrm{C}}}
$$

where $n_{\mathrm{D}}, n_{\mathrm{F}}$ and $n_{\mathrm{C}}$ are the respective RIs at 589, 486 and $656 \mathrm{~nm}$. Generally, an organic polymer with a high RI also displays a high dispersion (or low $v_{\mathrm{D}}$ ) and vice versa. Thus, poly(vinylcarbazole), for example, shows a high RI (1.675) and a low $v_{\mathrm{D}}$ (19), whereas Teflon or poly(tetrafluoroethylene) shows a low RI (1.345) and a high $v_{\mathrm{D}}(83) .{ }^{13}$ Evidently, development of polymeric materials with both high RI and $v_{\mathrm{D}}$ values (hence low optical dispersions) is a much sought after research goal.

Thanks to their highly polarizable $\pi$-electrons, $h b$-PTEPA(Co) and $h b$-PTEPA(Co)-UV both fulfil the requirement of exhibiting

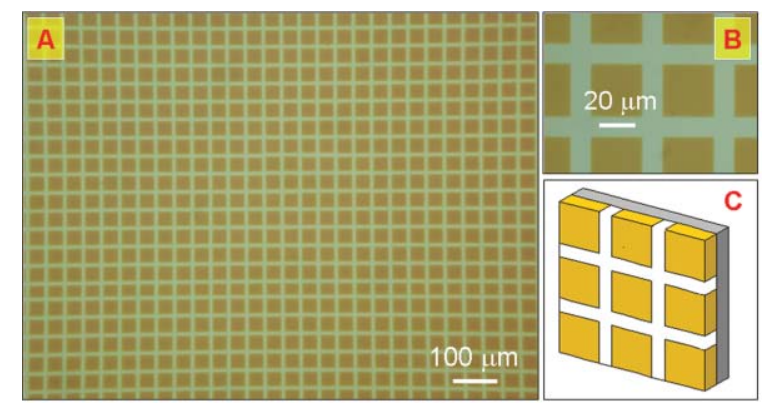

Fig. 1 (A) Optical micrograph of a two-dimensional photonic pattern generated by UV irradiation of $h b$-PTEPA(Co) through a $\mathrm{Cu}$-negative mask, (B) image with a high magnification, and (C) model of the photopattern, with brown and white colors denoting unexposed and exposed areas, respectively.

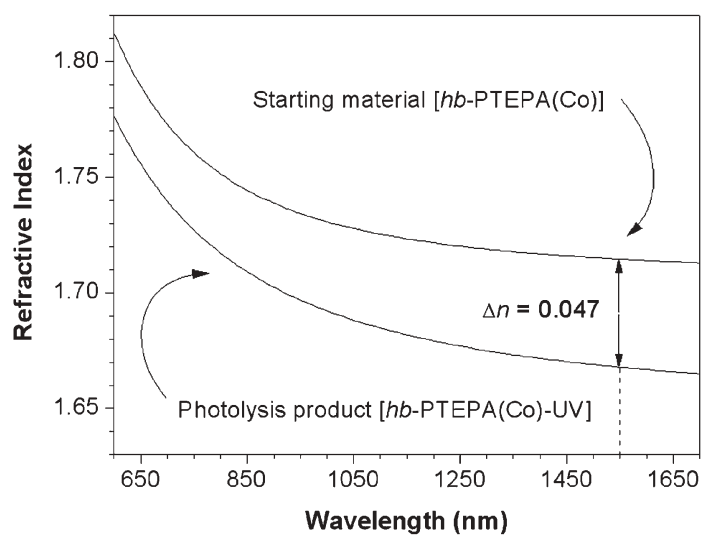

Fig. 2 Refractive indexes of thin films of the cobalt-containing polymer before $[h b$-PTEPA $(\mathrm{Co})]$ and after photolysis $[h b$-PTEPA(Co)-UV].

Table 1 Refractive indexes $(n)$ and revised Abbé numbers $\left(v_{\mathrm{D}}^{\prime}\right)$ of films of the cobalt-containing hyperbranched polymer before $[h b$-PTEPA(Co)] and after photolysis [ $h b$-PTEPA(Co)-UV]

\begin{tabular}{lllll}
\hline Polymer & $n_{1064}$ & $n_{1319}$ & $n_{1550}$ & ${ }^{v_{\mathrm{D}}{ }^{\prime}}$ \\
\hline$h b$-PTEPA(Co) & 1.727 & 1.718 & 1.715 & 59.8 \\
$h b$-PTEPA(Co)-UV & 1.687 & 1.674 & 1.668 & 35.5 \\
\hline
\end{tabular}

high RI values. Both of the polymers, like other conjugated polymers, ${ }^{14}$ absorb in the visible spectral region, showing higher RI values in the shorter wavelength regions. This interference not only complicates the RI measurements below $700 \mathrm{~nm}$ but also makes the associated low $v_{\mathrm{D}}$ values meaningless as they are derived from the resonance-enhanced, highly dispersive RI values.

To explore the real potential of optical applications of the polymers, it has been suggested to use a revised Abbé number $\left(v^{\prime}\right)$, utilizing their RI values at nonabsorbing wavelengths at 1064, 1319 and $2500 \mathrm{~nm} .{ }^{14}$ The first two wavelengths are chosen in view of the practical interest of commercial laser lines (Nd:YAG lasers), ${ }^{14 a, 15}$ while the last wavelength represents the longest measurable nonresonant line. The value at $2500 \mathrm{~nm}$ may well reflect the real optical dispersion but for more common and convenient usage, we suggest employing the RI value at $1550 \mathrm{~nm}$ to calculate $v_{\mathrm{D}}{ }^{\prime}$ values for conjugated polymers. ${ }^{16 c}$ The $v_{\mathrm{D}}{ }^{\prime}$ is thus now defined by equation 2 :

$$
v_{\mathrm{D}}^{\prime}=\frac{n_{1319}-1}{n_{1064}-n_{1550}}
$$

where $n_{1319}, n_{1064}$ and $n_{1550}$ are the RI values at 1319,1064 and $1550 \mathrm{~nm}$, respectively.

The $v_{\mathrm{D}}$ ' value for $h b$-PTEPA $(\mathrm{Co})$ is as high as 60 , while its photolyzed product of $h b$-PTEPA(Co)-UV exhibits a $v_{\mathrm{D}}{ }^{\prime}$ value of 36 (Table 1). Previously reported $v_{\mathrm{D}}{ }^{\prime}$ values for other conjugated polymers with absorptivity in the visible region have been from 9 to 38 , with the majority of the data in the range of $10-20 .{ }^{14 a}$ The $v_{\mathrm{D}}{ }^{\prime}$ values of our polymers are significantly higher, indicative of their lower optical dispersions and hence lower chromatic aberrations. Together with their very high RI values, our polymers are promising candidate materials for applications in the construction of photonic and holographic devices such as readonly memories in the near-IR region. ${ }^{16}$

Metallic species such as iron, nickel and cobalt nanoclusters are known to catalyze CNT growth in the chemical vapor deposition 


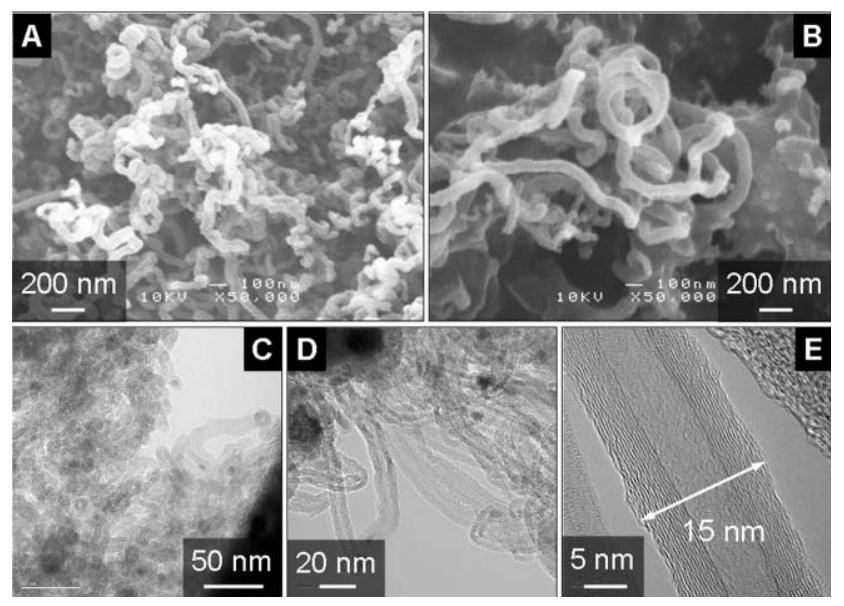

Fig. 3 (A and B) SEM and (C-E) TEM microphotographs of the carbon nanotubes prepared by a CVD process at $700{ }^{\circ} \mathrm{C}$ on silicon wafers spin-coated with thin films of $h b$-PTEPA(Co).

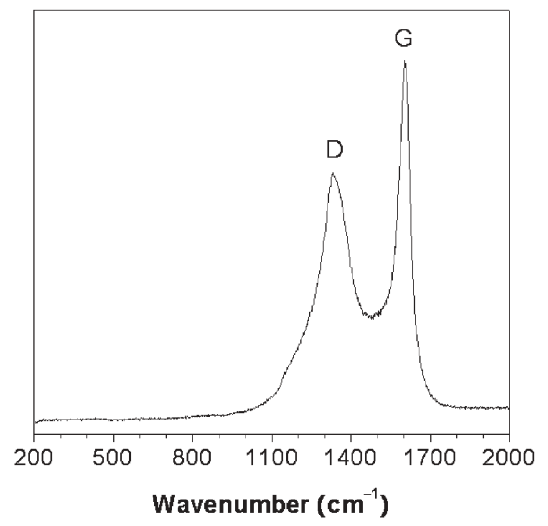

Fig. 4 Raman spectra of multiwalled carbon nanotubes fabricated by the CVD process catalyzed by the cobalt nanoclusters generated in-situ from pyrolysis of the $h b$-PTEPA(Co) film.

(CVD) process. ${ }^{17}$ Because of the ready thermal curability of the $h b$-PDYs ${ }^{8}$ spin-coated films of $h b$-PTEPA(Co) are expected to restrict the agglomeration of the cobalt nanoclusters formed in situ in the CVD process and hence to provide nanoscopic catalyst seeds for the CNT growth. This proves to be the case. Uniform bundles of CNTs are grown by the CVD process at $700{ }^{\circ} \mathrm{C}$ with acetylene gas as the carbon source (Fig. 3). As expected from a CNT growing temperature below $800{ }^{\circ} \mathrm{C},{ }^{18}$ the formed CNTs are multiwalled in nature. Thanks to the homogeneous organometallic thin films, the CNTs have a narrow size distribution or a small dispersity, with the diameters of their external walls being as small as $15 \mathrm{~nm}$ (Fig. 3E).

Raman spectra of the CNTs (Fig. 4) reveal two characteristic peaks: a sharp $\mathrm{G}$ band at $\sim 1600 \mathrm{~cm}^{-1}$, which corresponds to a first-order Raman process involving an in-plane oscillation of the carbon atoms in the graphene sheet, and a relatively broad $\mathrm{D}$ band at $\sim 1340 \mathrm{~cm}^{-1}$, which relates to the second-order defect-induced Raman mode involving a one phonon elastic scattering process. The appearance of these two Raman bands is in good agreement with the multiwalled nature of the CNTs, as proved by TEM analysis.
In conclusion, the cobalt-complexed $h b$-PDY possesses a good film formability and shows a high optical transparency in the long wavelength region. UV irradiation of the brownish $h b$-PTEPA(Co) film induces photobleaching, accompanied by large changes in its RI values. The organocobalt polymer and its UV-cured product exhibit large $v_{\mathrm{D}}$ ' values hence low optical dispersions in the nearIR region. The thin film of $h b$-PTEPA $(\mathrm{Co})$ can efficiently catalyze the growth of CNTs in the CVD process, making it a versatile material for an array of high-tech applications.

The work described in this paper was partially supported by the Research Grants Council of Hong Kong (602706, HKU2/05C, 603505 and 603304), the National Science Foundation of China (20634020), and the Ministry of Science and Technology of China (2002CB613401). B. Z. T. is grateful for support from the Cao Guangbiao Foundation of Zhejiang University.

\section{Notes and references}

1 A. S. Abd-El-Aziz, Macromol. Rapid Commun., 2002, 23, 995.

2 S. A. Jenekhe and J. A. Osaheni, Science, 1994, 265, 765.

3 (a) G. R. Newkome, E. He and C. N. Moorefield, Chem. Rev., 1999, 99, 1689; (b) D. Astruc and F. Chardac, Chem. Rev., 2001, 101, 2991.

4 Organic polymers usually show RIs in the range of 1.338-1.71: J. C. Seferis, in Polymer Handbook, ed. J. Brandrup and E. H. Immergut, Wiley, New York, 3rd edn, 1989, p. VI/451.

5 (a) M. Olshavsky and H. R. Allcock, Macromolecules, 1997, 30, 4179; (b) C. Paquet, P. W. Cyr, E. Kumacheva and I. Manners, Chem. Commun., 2004, 234.

6 (a) P. K. H. Ho, S. Thomas, R. H. Friend and N. Tessler, Science, 1999, 285, 233; (b) H. Kudo, M. Yamamoto and T. Nishikubo, Macromolecules, 2006, 39, 1759; (c) T. Höfler, M. Weinberger, W. Kern, S. Rentenberger and A. Pogantsch, Adv. Funct. Mater., 2006, 16, 2369; (d) C. Bertarelli, A. Bianco, F. D'Amore, M. C. Gallazzi and G. Zerbi, Adv. Funct. Mater., 2004, 14, 357; (e) Y. Kato and K. Horie, Macromol. Chem. Phys., 2002, 203, 2290; (f) Q. Lin, B. Yang, J. Li, X. Meng and J. Shen, Polymer, 2000, 41, 8305.

7 (a) H. Dong, J. W. Y. Lam, M. Häussler, R. Zheng, H. Peng, C. C. W. Law and B. Z. Tang, Curr. Trends Polym. Sci., 2004, 9, 15; (b) M. Häussler and B. Z. Tang, Adv. Polym. Sci., 2007, 209, DOI: 10.1007/ 12_2007_112.

8 M. Häussler, R. Zheng, J. W. Y. Lam, H. Tong, H. Dong and B. Z. Tang, J. Phys. Chem. B, 2004, 108, 10645.

9 M. Häußler, J. W. Y. Lam, R. Zheng, H. Dong, H. Tong and B. Z. Tang, J. Inorg. Organomet. Polym. Mater., 2005, 15, 519.

10 W. Y. Chan, A. Berenbaum, S. B. Clendenning, A. J. Lough and I. Manners, Organometallics, 2003, 22, 3796.

11 W. F. Edgell and J. Lyford, IV, J. Chem. Phys., 1970, 52, 4329.

12 (a) M. Campbell, D. N. Sharp, M. T. Harrison, R. G. Denning and A. J. Turberfield, Nature, 2000, 404, 53; (b) L. Wu, W. Y. Y. Tong, Y. Zhong, K. S. Wong, J. Hua, M. Häußler, J. W. Y. Lam and B. Z. Tang, Appl. Phys. Lett., 2006, 89, 191109.

13 H. U. Simmrock, A. Marthy, L. Dominguez, W. H. Meyer and G. Wegener, Angew. Chem., Int. Ed. Engl., 1989, 28, 1122.

14 (a) C. J. Yang and S. A. Jenekhe, Chem. Mater., 1994, 6, 196; (b) C. J. Yang and S. A. Jenekhe, Chem. Mater., 1995, 7, 1276.

15 D. Y. Kim, M. Sundheimer, A. Otomo, G. I. Stegman, H. G. H. Winfried and G. R. Mohlhaupt, Appl. Phys. Lett., 1993, 63, 290.

16 (a) E. Hendrickx, C. Engels, M. Schaerlaekens, D. Van Steenwinckel, C. Samyn and A. Persoons, J. Phys. Chem. B, 2002, 106, 4588; (b) C. Lu, C. Guan, Y. Liu, Y. Cheng and B. Yang, Chem. Mater., 2005, 17, 2448; (c) A. J. Miller, S. W. Nam, J. M. Martinis and A. V. Sergienko, Appl. Phys. Lett., 2003, 83, 791.

17 (a) A. K. Chatterjee, M. Sharon, R. Baneriee and M. NeumannSpallart, Electrochim. Acta, 2003, 48, 3439; (b) Z. P. Huang, D. Z. Wang, J. G. Wen, M. Sennett, H. Gibson and Z. F. Ren, Appl. Phys. A: Mater. Sci. Process., 2002, 74, 387.

18 J. Q. Lu, T. E. Kopley, N. Moll, D. Roitman, D. Chamberlin, Q. Fu, J. Liu, T. P. Russell, D. A. Rider, I. Manners and M. A. Winnik, Chem. Mater., 2005, 17, 2227. 